\title{
SUR UN CAS DE GALE NORVÉGIENNE
}

\author{
Par Amadeu FIALHO
}

Il y a quelques mois, l'auxiliaire d'un service scientifique nous apporta un fragment de peau d'un cadavre formolisé, nous demandant de l'examiner afin de déceler la nature des lésions diffuses si curieuses que présentait ce cadavre, à tel point que les étudiants se refusaient de l'utiliser pour leurs travaux anatomiques.

Notre surprise a été grande, lorsque nous avons vu qu'il s'agissait d'un cas de gale, avec abondance de parasites et prolifération des plans superficiels de l'épiderme, constituant une épaisse couche, dont nous ferons l'étude ultérieurement. Quelques jours après, nous avons obtenu de plus grands fragments de peau, et nous avons constaté qu'il s'agissait d'un cas de gale norvégienne ou croûteuse. De très petits morceaux des régions épaissies, soumises à l'action de la lessive de soude, laissaient voir une grande abondance d'acariens à tous les stades de leur développement.

Lorsque le diagnostic de gale croûteuse fut établi, nous avons essayé d'obtenir des renseignements sur le cas en question et l'autorisation de faire l'autopsie.

Il s'agissait d'une femme pauvre, atteinte d'une tuberculose grave, dans un état de dénutrition accentué, qui habitait un tertre à Nicteroy et qui entra à l'hôpital public de cette ville. En peu de temps, elle succombait à la bacillose. Lorsqu'elle entra dans la clinique, on constata la présence des lésions cutanées. Elle se plaignait de prurit intense et diffus. Pendant son séjour à l'hôpital, aucun cas de gale n'a été constaté.

Ayant obtenu la permission d'examiner le cadavre, nous avons pratiqué l'autopsie complète, dont le protocole est le suivant :

\section{Autopsie $n^{0} 44\left(4^{e}\right.$ série $)$}

Examen externe: Cadavre de femme métisse, régulièrement conformée, en dénutrition ( $\mathrm{pl} . \mathrm{X}, 1$ ).

Ayant été préalablement injecté au formol, ce cadavre présente une rigidité caractéristique. La macération qu'il a subi dans le

Annales de Parasitologie, T. XII, N ${ }^{\circ} 6 .-1$ er novembre 1934 , p. 472-481. 
liquide conservateur modifia certaines particularités du tégument cutané, mais, d'une manière générale, l'examen est encore possible.

Ce qui frappe d'abord l'attention, ce sont des formations croûteuses, brunes, qu'on observe surtout à la tête (fig.) et au trone, bien qu'elles soient encore visibles sur certaines parties des membres. La tête porte de courts et rares cheveux. Un dépôt croûteux châtain-fon-

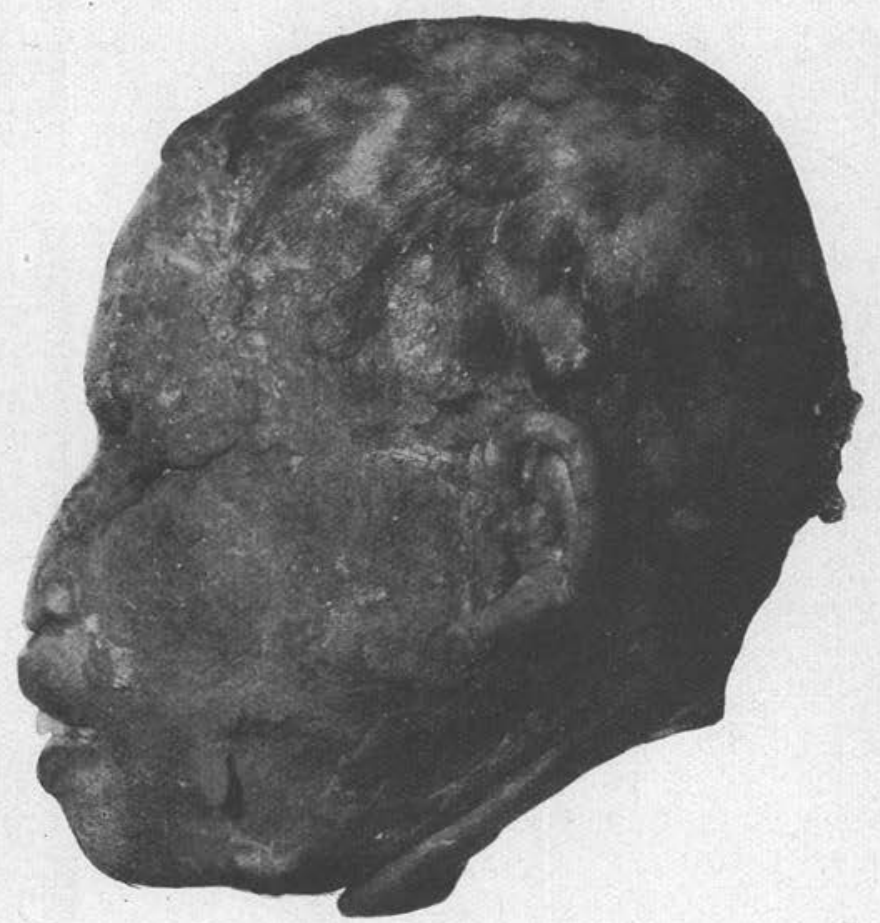

Fı́. - Tête d'une femme métisse atteinte de gale norvégienne.

cé atteignant en quelques points l'épaisseur de $2 \mathrm{~mm}$., recouvre tout le cuir chevelu ; il peut être détaché avec des pinces en morceaux ou en petites lames. Aux régions frontale, temporales et occipitale, les croûtes s'épaississent.

Les téguments sont altérés sur toutes les parties du visage, avec plus d'intensité aux régions malaires et aux oreilles. Le dépôt croûteux s'étend du pavillon de l'oreille au conduit auditif. A la région zygomatique, on trouve des formations, avec une épaisseur 
de $2 \mathrm{~mm}$. à moitié détachées par macération. Au front, les croûtes s'entassent et envahissent la bande des sourcils. La face externe des bras montre les mêmes altérations. La région mastoïdienne, les coudes et la face postérieure des avant-bras sont également envahis. Les mains le sont aussi, mais les altérations qu'on y observe sont due surtout à la macération de l'épiderme. Autour des ongles, il n'y a pas de dépôt de croûtes, mais de petites lésions.

A la région mammaire, des deux côtés, aux flanes et à la face postérieure du tronc, les croûtes sont entassées en abondance, et atteignent aux flancs l'épaisseur de près de 3 à $4 \mathrm{~mm}$. La région du pubis et les grandes lèvres, l'orifice du vagin et la partie antérieure de ce canal présentent aussi des formations croûteuses. Les mêmes lésions s'étendent sur les membres inférieurs, surtout à leur face externe, atteignant les pieds, où elles sont moins accentuées.

Examen interne : Encéphale sans altérations ; appareil circulatoire sans particularité. Les deux poumons présentent de petits nodules de caséification, surtout à droite. La rate et le foie ont une coloration brune, sans modification de forme ni de volume. Les reins sont normaux et leur surface est lisse. Aucune particularité dans l'utérus, l'ovaire et les trompes.

Examen microscopique : Les croûtes, retirées du cadavre, montrent un nombre considérable de Sarcoptes scabiei Linné, à tous les stades de développement depuis l'œuf jusqu'aux formes adultes des deux sexes.

La moelle osseuse se présente en activité hématopoiétique. En beaucoup de points, nous avons pu constater la présence d'éléments éosinophiles.

La rate présente des polymorphonucléaires en quantité appréciable.

Les ganglions lymphatiques ont subi des altérations inflammatoires aiguës évidentes.

Le foie présente une infiltration graisseuse diffuse.

Le poumon est atteint de tuberculose exsudative bronchopneumonique avec ses altérations habituelles.

Les reins présentent des lésions diverses. Il existe çà et là des cylindres. A ces altérations de dégénérescence s'associent des altérations inflammatoires, sous la forme de petits foyers constitués surtout par des lymphocytes et des plasmazellen. Ils sont surtout localisés aux parties superficielles de la couche corticale.

La peau est la plus altérée ; on observe de la parakératose exu- 
bérante, qui forme des couches épaisses. En effet, ainsi que nous l'avons déjà observé dans de nombreux autres cas, nous retrouvons l'aspect particulier de la gale norvégienne, où la formation de croûtes est dùe plutôt à la parakératose qu'à l'hyperkératose. C'est ainsi qu'aux points où les lésions cutanées prennent la plus grande épaisseur, les cellules lamineuses de la couche superfícielle présentent des noyaux dans toute son épaisseur. Toutefois, il n'y a pas absence absolue de cellules kératinisées.

Les éléments cellulaires ne sont pas disposés sur une seule couche, mais forment un curieux système de canaux, qui suivent une direction parallèle à la peau dans des plans superposés et qui s'anastomosent. Cette disposition a été comparée par quelques auteurs aux galeries d'une mine (pl. X, 2).

Les canaux profonds sont plus larges et c'est dans ces nombreux tunnels que se trouvent les acariens à toutes les phases de leur développement. Généralement, les formes adultes se trouvent dans les cavités les plus profondes. On voit aussi, dans certaines galeries, une substance albuminoïde coagulée, ayant l'aspect d'une masse rose légèrement granuleuse avec les colorants habituels. Dans d'autres galeries, les œufs et les jeunes formes parasitaires se rangent en série. On trouve aussi les excréments des acariens, dont la quantité est variable suivant les endroits. Il y a des points où l'on observe un dépôt granuleux et basophile même à un faible grossissement.

En utilisant la méthode de Gram, on met en évidence un nombre considérable de microbes, doublant les galeries, qui environnent les parasites et formant des amas tantôt répandus à la surface des croûtes, tantôt situés plus profondément jusqu'au contact de l'épiderme.

Le corps muqueux est d'épaisseur variable. Mais cette couche, en général mince et parfois même très réduite, s'épaissit en quelques points, sans jamais atteindre une épaisseur exagérée. Les prolongements qu'elle forme s'enfoncent dans le derme en cônes minces ou en formations plus épaisses (pl. XI, 3). Aux points où le corps muqueux est le plus développé, les cellules prennent un aspect particulier, clair, en donnant tantôt l'impression d'éléments hydropiques, tantôt celle de vacuoles. Mais nous insistons sur le fait que l'épiderme est peu développé et même fréquemment atrophié. Son altération prédominante est la parakératose. La couche basale est irrégulièrement pigmentée, bien qu'il s'agisse d'une mûlatresse. On observe souvent une séparation entre le derme et l'épiderme, causée par de l'œdème (exosérose) (pl. XI, 4). En effet, cet œdème est une altération commune dans toutes les 
préparations observées et peut être trouvé à tous les degrés. Les peites veines sont dilatées et quelques-unes sont rompues, avec des suffusions hémorragiques à la périphérie. En de rares points, cet épanchement sanguin infiltre même l'épiderme. De telles hémorragies, de même que l'œdème, semblent avoir été très fréquentes au cours̀ de la maladie. Ces hémorragies sont mises en évidence par la grande quantité de cellules chargées d'hémosidérine, trouvées dans le derme. Quant à l'œdème, il doit être représenté par les liquides albumineux trouvés dans les galeries épidermiques, dont nous avons déjà fait mention. Kiess avait raison quand il montra le manque de précision de l'expression d'Unna, qui définit les altérations de la gale norvégienne comme * einen trockenen Oberhautkatarrh ».

Il y a des observateurs, tels qu'Ijiri et Dubreuilh, qui n'admettent pas une telle opinion. Il semble que, selon les individus, il peut se développer une forme sèche ou humide de telle ou telle parasitose (as ich bi dem einen Individum eine trockene, bei dem andere eine feuchte Form der norwegischen Kr'atze entwickelt). Cette assertion est justifiée par la présente observation, car nous constatons la présence de sérosité d'œdème qui s'étend du derme jusqu'aux galeries parasitaires, situées dans les couches de parakératose. Outre l'œdème de la vaso-dilatation et les hémorragies, nous trouvons encore dans le derme des infiltrations périvasculaires abondantes, avec lymphocytes, quelques plasmazellen et mastzellen. Ces derniers éléments sont relativement abondants en quelques points. Nous avons constaté aussi la présence de polymorphonucléaires en nombre très variable. On trouve même des micro-abcès en formation dans quelques préparations. Il n'y a pas d'éosinophiles. Les fibres élastiques sont un peu raréfiées. Toutes les altérations que nous venons de décrire se rencontrent dans les plans supérieurs du derme.

Les plans plus profonds, dans le voisinage du tissu sous-cutané, se présentent normalement, excepté en certains points du tégument mammaire, dans le voisinage du mamelon. Là, il existe, même profondément, un processus inflammatoire, diffus, avec infiltration périvasculaire. Les canaux galactophores sont aussi altérés.

En comparant histologiquement les fragments recueillis en divers points du revêtement cutané et les aires de transition cutanomuqueuses, nous avons remarqué ce qui suit :

Au niveau de l'auréole mammaire, il y a formation de croûtes suivie de lésions peu accentuées de l'épiderme; mais le derme présente un processsus inflammatoire diffus, qui gagne en profondeur, en quelques points, ainsi que nous l'avons déjà dit. Nous 
avons trouvé dans le corion des chromatophores abondants et quelques cellules avec de l'hémosidérine.

Aux cuisses et aux membres supérieurs, les altérations épidermiques sont plus remarquables et les lésions du derme sont plus intenses. Nous y avons remarqué un fort œdème, l'infiltration inflammatoire du derme et un dépôt d'hémosidérine. Au cuir chevelu, les altérations sont diffuses et accentuées, avec atrophie de la couche épithéliale et de ses dérivés. Les parasites s'y trouvent en abondance. On trouve une particularité dans la région axillaire, c'est l'hyperkératose, bien qu'elle ne soit pas très intense (Pl. XII, 5). Les localisations parasitaires sont en petit nombre et nous pouvons vérifier que, dans les points où se logent les acariens, les cellules épidermiques deviennent volumineuses et claires, en ébauchant tout de suite la parakératose. En même temps apparaissent les altérations du derme (pl. XII, 5 et 6 ).

Au bord de l'orifice anal et dans le vagin, nous avons trouvé une infiltration cellulaire du chorion, caractéristique d'un processus inflammatoire chronique. L'épithélium présente une discrète hyperplasie et un certain degré de dissociation cellulaire. On n'y trouve pas de parasites. Dans le conduit auditif, on trouve les mêmes altérations. Les parasites y sont aussi présents.

Parasites : Ce qui a frappé notre attention, au premier examen, ce fut la richesse en acariens (pl. XIII, 7). C'est cette abondance de parasites et la formation de croùtes qui caractérisent la gale norvégienne.

Après de nombreux essais, nous avons utilisé l'action prolongée de la lessive de soude et nous avons obtenu la désagrégation complète des lames croûteuses, mais les parasites sont devenus tellement friables qu'une compression, même légère, déterminait la destruction du squelette chitineux. Le diaphanol rend aussi de réels services, mais il fait perdre aux éléments parasitaires leur coloration.

A l'examen microscopique, on constate une grande prédominance des femelles. Dans quelques préparations, nous avons trouvé un plus grand nombre de mâles, mais exceptionnellement. Sur toutes les lames, on observe toutes les formes parasitaires : adultes, nymphes, larves et œufs, embryonnés ou non. Les fèces sont abondantes (pl. XIII, 7).

Les observations brésiliennes, aussi bien que les observations étrangères, ne consignent pas les mesures de ces acariens. Nous avons profité de notre matériel pour procéder à des mensurations et nous avons obtenu les chiffres suivants, exprimés en $\mu$. 


\begin{tabular}{|c|c|c|}
\hline & CEuf & $162 \times 99$ \\
\hline & Euf avec larve ........... & 162 à $171 \times 99$ \\
\hline & Larve hexapode ............. & $162 \times 117$ \\
\hline ter & mâle..... & $252 \times 171$ \\
\hline & femelle ... & $392 \times 315$ \\
\hline
\end{tabular}

Ces chiffres sont très rapprochés, non seulement de ceux que nous avons trouvé dans d'autres cas de gale norvégienne, mais encore de ceux qui concernent le sarcopte de la gale commune.

Dimensions de Sarcoptes scabiei var. hominis, d'après César Pinto.
CEuf
$150 \times 100$
Larve hexapode ................................ 1400

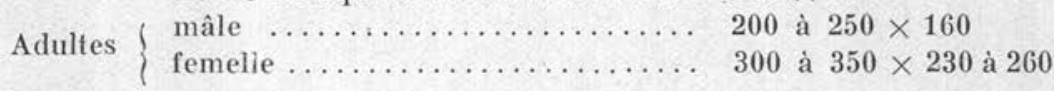

Les mensurations relatées par divers auteurs, que nous avons trouvées dans l'ouvrage de Kiess, sont les suivantes :

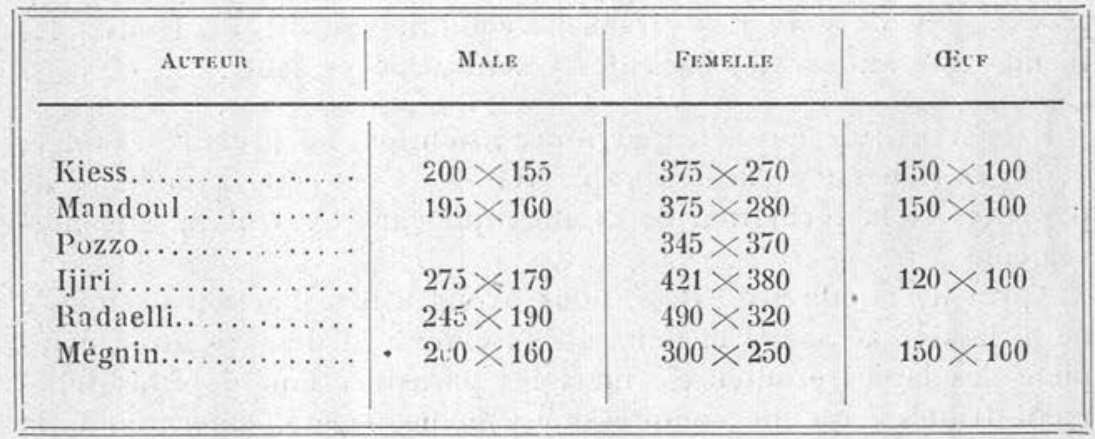

Les larves hexapodes (pl. XIII, 8) mesurent, d'après Buxton, $150 \times 100 \mu$ et, d'après Cameron, $163 \times 177 \mu$. Ces dernières dimensions concordent absolument avec celles que nous avons enregistrées : $162 \times 177 \mu$. La diversité des dimensions peut être expliquée en partie par les altérations dues aux liquides employés pour l'étude de ces acariens.

La morphologie générale des parasites est celle que l'on trouve décrite dans tous les traités classiques de parasitologie (pl. XIV, 9).

RELATION des paRAsites AVEc Les tissus. - D'une manière générale, les parasites se trouvent en plus grande abondance dans les couches profondes des croûtes (pl. XIV, 10). 
Les œufs sont généralement observés en série. Les formes adultes sont souvent en rapport avec l'épiderme. L'examen de nos préparations nous a permis d'observer la formation de tunnels ou de galeries dans l'épiderme, généralement entourées de cellules en parakératose. Quelquefois même, les parasites arrivent au derme, mais ils en sont le plus fréquemment séparés par une ou deux couches de celiules épithéliales. Chez quelques acariens, situés profondément, nous avons observé une tuméfaction de l'une ou de l'autre cellule de la cavité gastrique (Ricksttsia ?).

Autour du corps des parasites, nous avons toujours constaté une grande quantité de germes, quelquefois suspendus dans la sérosité de l'œdème.

\section{Discussion}

Notre cas a été minutieusement étudié, particulièrement du point de vue histologique. Les altérations cutanées, très intéressantes, sont variables d'intensité d'un endroit à l'autre, mais elles peuvent être résumées ainsi : parakératose, œdème inter- et intracellulaire, hyperhémie et œdème du derme, infiltration périvasculaire et formation de petits foyers inflammatoires plus ou moins aigus, avec réaction leucocytaire.

De semblables lésions peuvent être observées, d'une façon plus nette, dans les régions où abondent les parasites. Néanmoins, c'est au niveau des localisations isolées des parasites que nous voyons s'ébaucher toutes ces réactions. Nous pouvons donc les étudier une à une.

C'est la présence de ces altérations initiales qui nous font supposer que le parasite est la cause de quelques-unes de ces lésions, moins par sa présence que par l'action des produits qui en dérivent tels que la sécrétion de ses glandes vénimeuses, ses fèces, etc.

La réaction insolite est causée par les conditions particulières des malades. Quelques auteurs, Ijiri et d'autres, ont invoqué l'existence d'une toxine pour expliquer même les lésions viscérales, telle que la néphrite, etc. Quoiqu'il n'y ait pas de répercussion si éloignée, l'hypothèse d'une substance toxique est tout à fait acceptable, ayant observé toutes les altérations qu'un acarien peut déterminer.

La présence d'une grande quantité de germes dans nos préparations montre quel degré atteint la richesse microbienne de ces croûtes et, par suite, les multiples possibilités des infections cutanées. De là, la lymphadénite chronique, avec ganglions périphériques qui existaient dans notre cas ainsi que dans d'autres. 
Dans notre observation, il n'y avait même pas de réactions aiguës produites par les Coccus. Nous n'avons pas remarqué d'éosinophilie locale. L'étude histologique des viscères a démontré une tuberculose en évolution et des altérations banales, sans rapport avec la parasitose cutanée. Dans le foie seulement, nous avons signalé un abcès microscopique. Nous pensons que cela est en relation avec une poussée infectieuse, déjà démontrée par les altérations ganglionnaires. Les lésions rénales trouvées sont de deux types : lésions de dégénérescence et lésions inflammatoires.

Bien que nous ne soyons pas spécialisés en parasitologie, il nous semble que le parasite de la gale norvégienne est le même que celui de la gale commune. Dans le cas du professeur Dubreuilh, le fait est éclairé par l'existence de la contagion de l'entourage: Les individus qui se sont infectés au contact de la porteuse de la gale norvégienne, ef parmi eux ses parents, ont contracté une gale commune.

L'argument de Buxton, rapporté par Costa Lima, a une certaine

\section{EXPLICATION DES PLANCHES}

Planche $\mathrm{X}$

Fig. 1. - Femme métisse atteinte de gale norvégienne.

Frg. 2. - Eléments cellulaires disposés sur une seule couche, formant un système de canaux qui suivent une direction parallèle à la peau dans des plans superposés et qui s'anastomosent.

\section{Planche XI}

Fı́. 3. - Galeries présentant des œufs et une substance albuminoïde coagulée. Frg. 4. - Séparation entre le derme et l'épiderme causée par l'œdème.

\section{Planche XII}

Frg. 5. - Hyperkératose de la région axillaire.

Fig. 6. - Altérations du derme.

\section{Planche XIII}

Fig. 7. - Eufs de Sarcoptes scabiei var. hominis.

Fic. 8. - Larves hexapodes de $S$. scabiei var. hominis.

\section{Planche XIV}

Frg. 9. - S. scabiei var. hominis, mâle et femelle.

Fia. 10. - Sarcoptes dans les couches profondes des croûtes. 


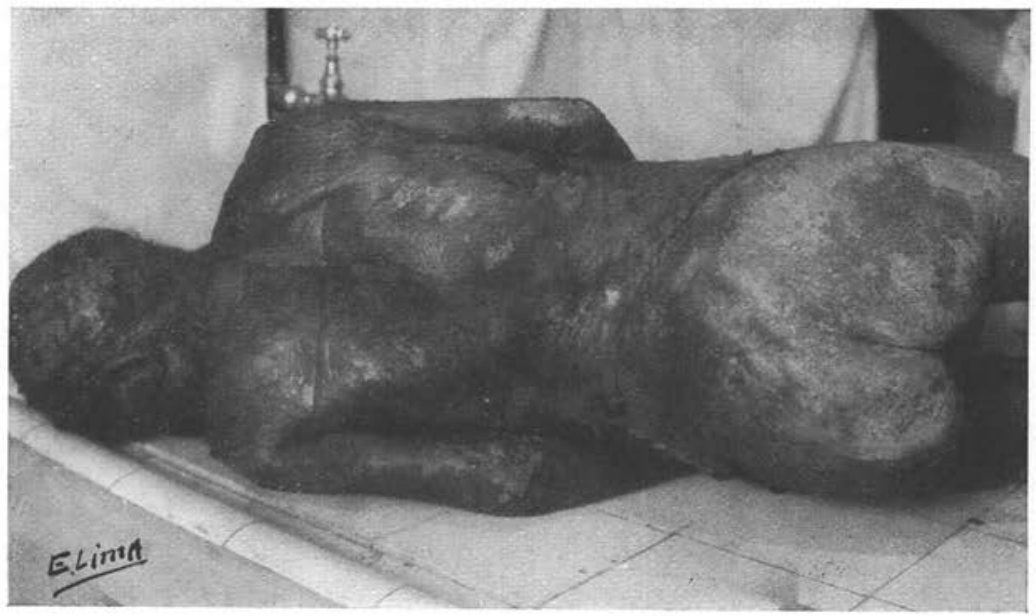

FIG. 1.

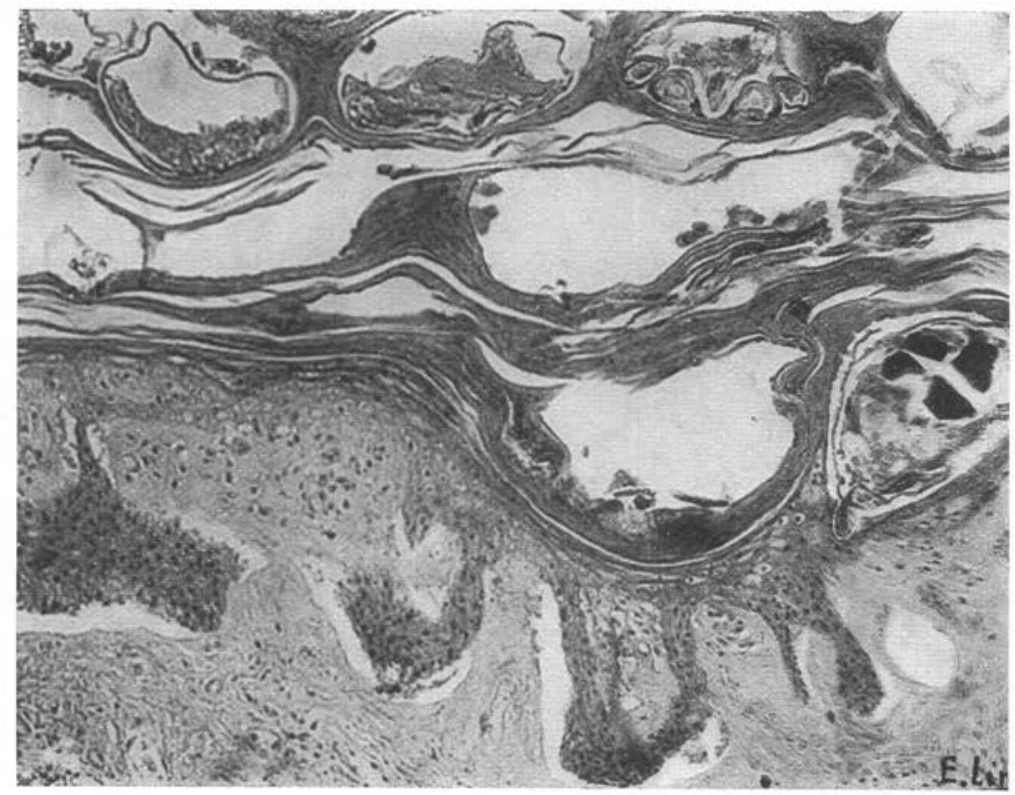

Fig. 2. 



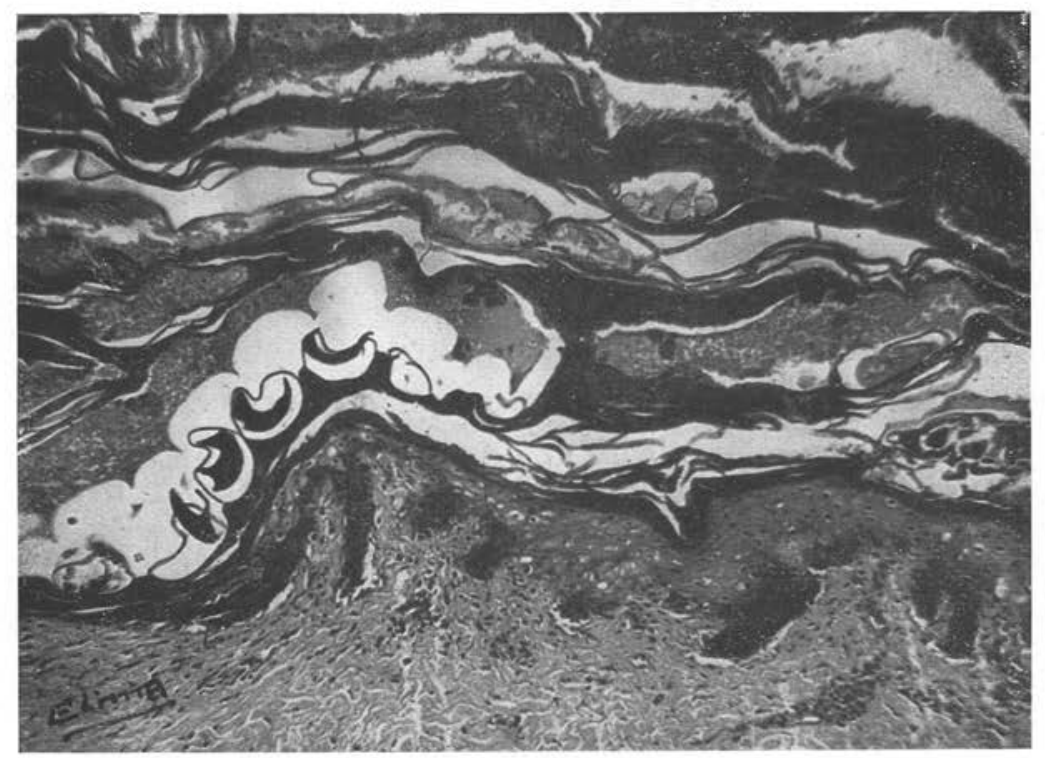

Fig. 3.

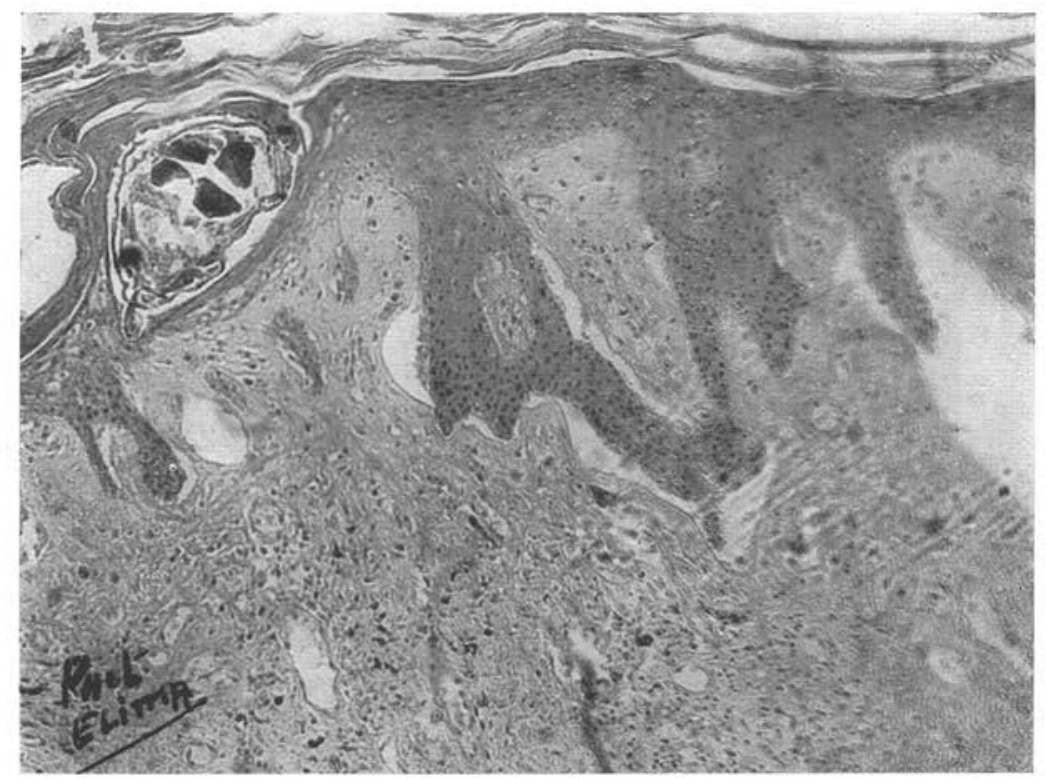

FIG. 4. 



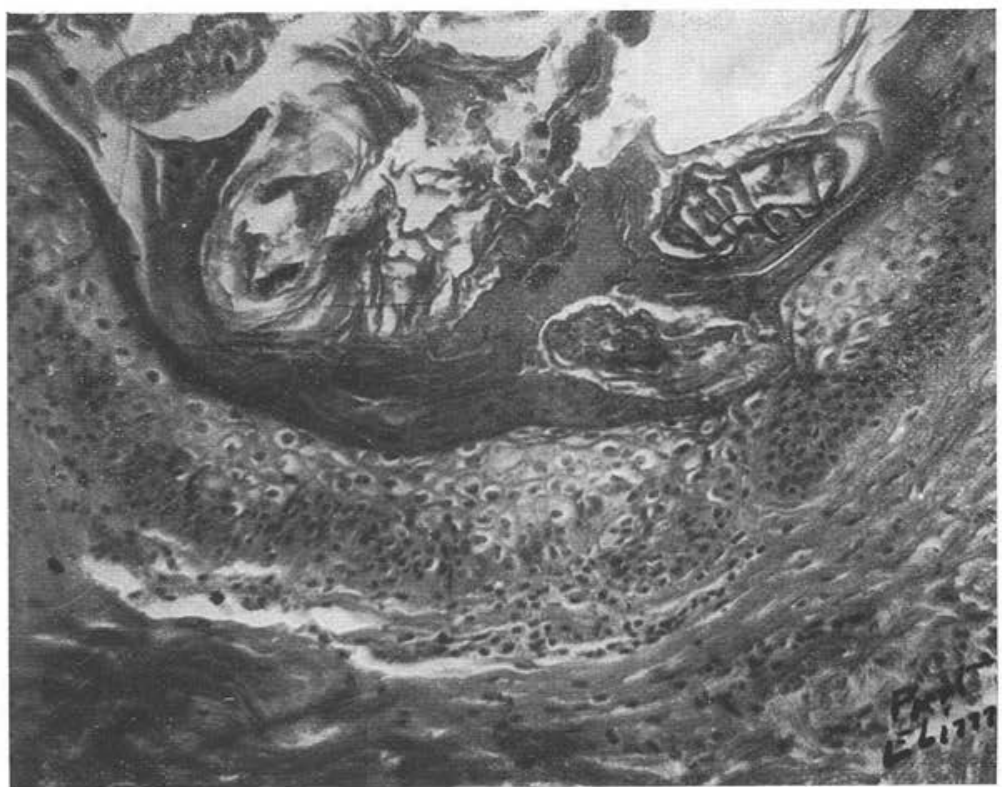

FIG. 5.

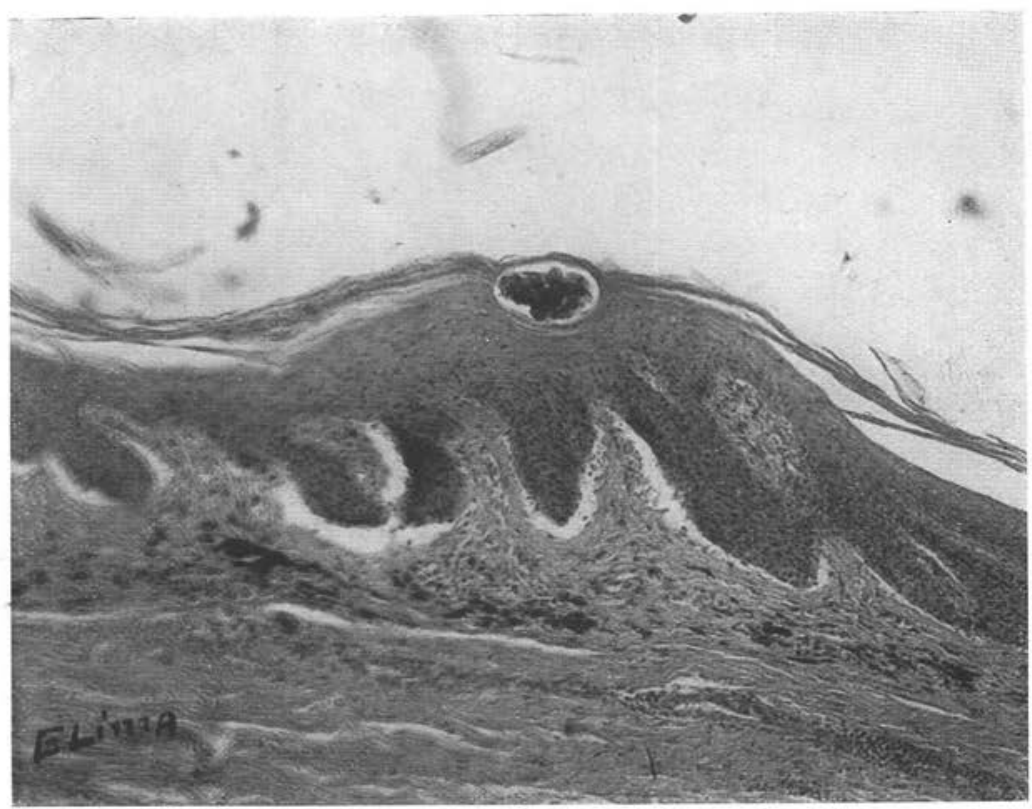

Fig. 6. 



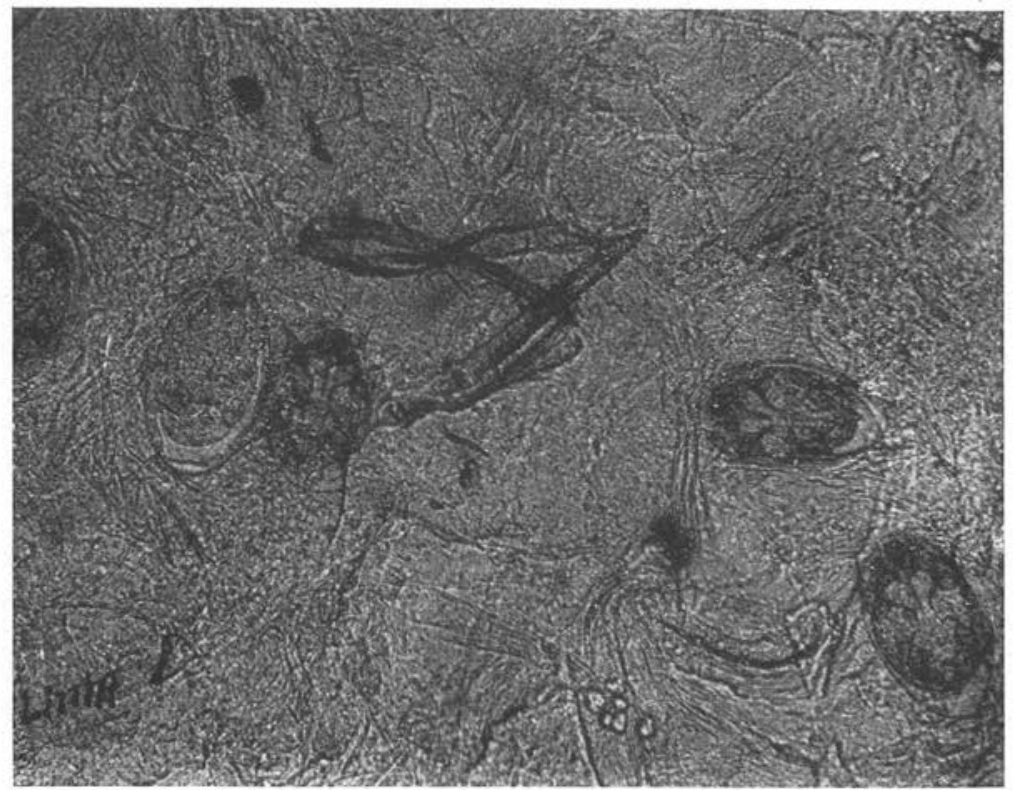

Fig. 7.

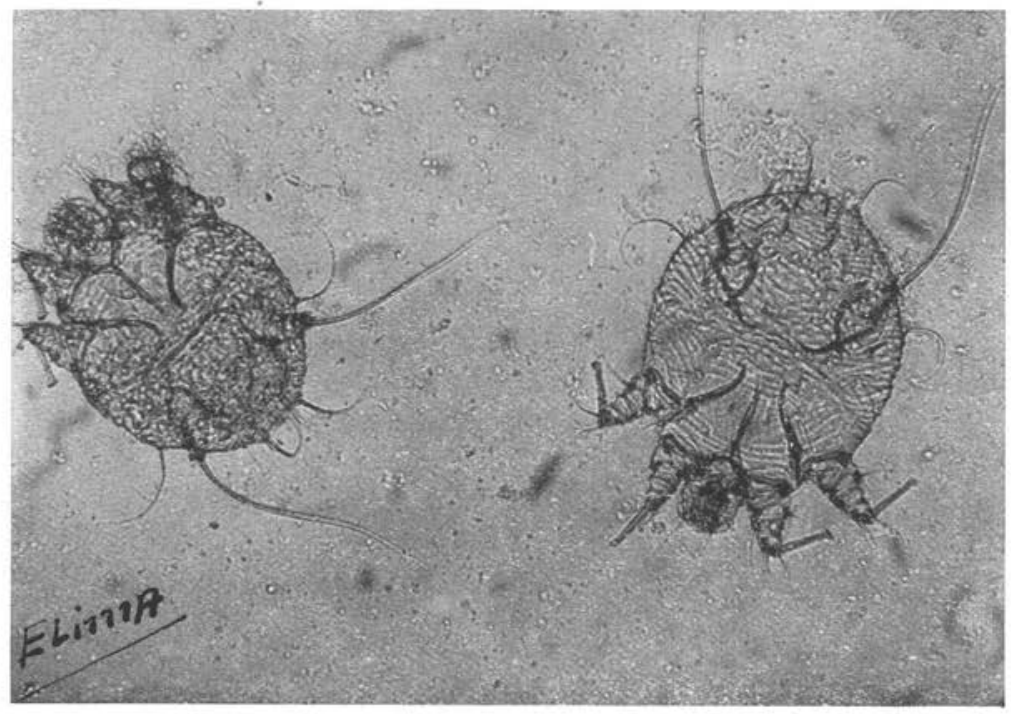

Fig. 8. 



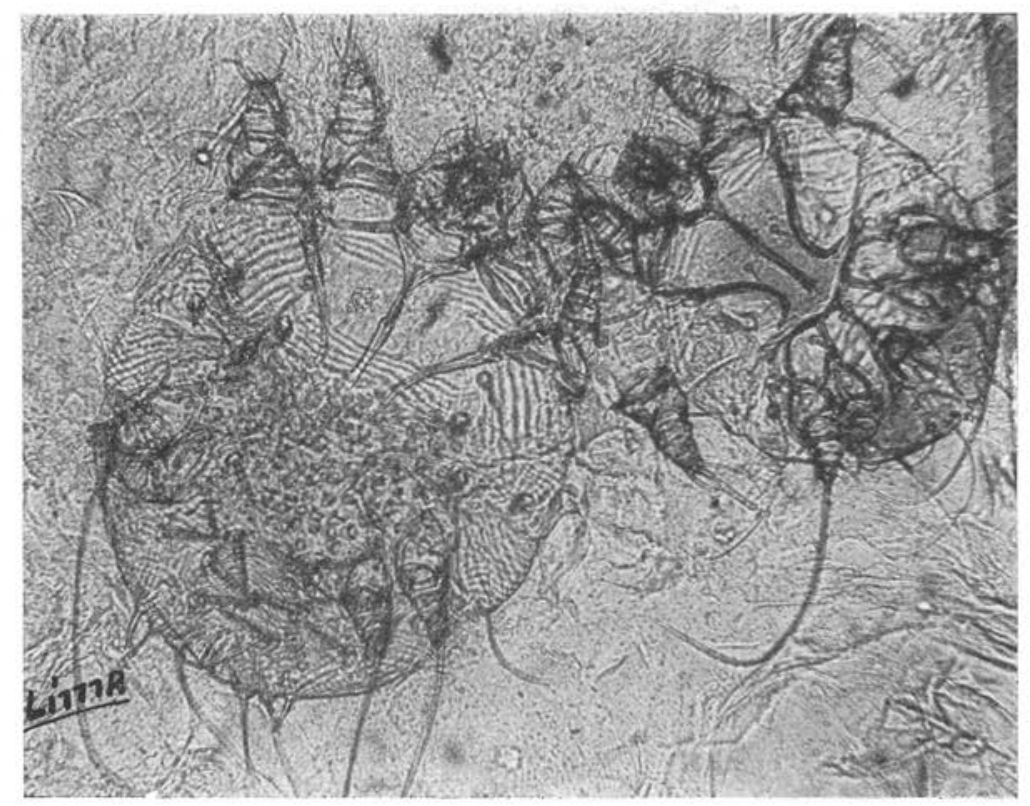

Fig. 9.

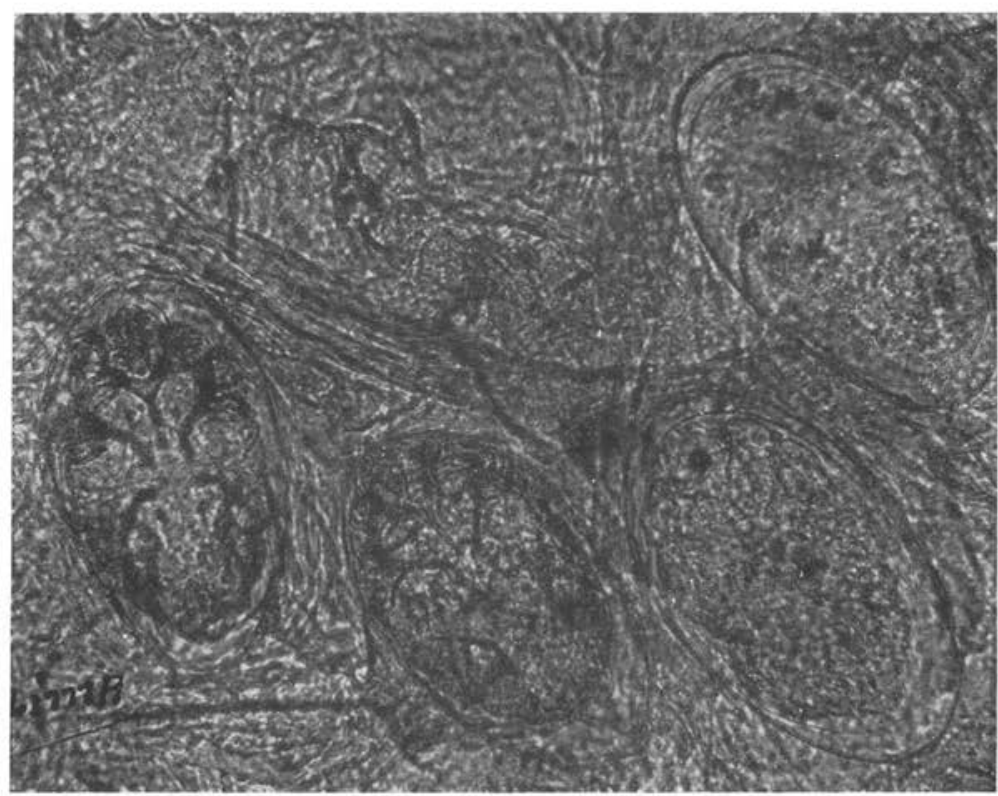

FiG. 10. 

valeur : Buxton, en argumentant en faveur de l'identité des deux formes, dit qu'étant donnée la rareté de la gale croûteuse, si l'acarien qui la détermine était une forme distincte de $S$. scabiei var. hominis, il serait éteint depuis longtemps. Cependant, les idées de Fürstenberg sur la variété des parasites ont trouvé des adeptes.

L'aspect spécial de la gale norvégienne est dû, vraisemblablement, aux conditions particulières des malades qui ont contracté leur affection dès l'enfance et l'ont conservée pendant plusieurs années. Tel est le cas rapporté par le $\mathrm{D}^{\mathrm{r}}$ Orsini de Castro : il s'agissait d'une femme, âgée de 30 ans, et atteinte de gale contractée dès l'enfance. Le malade de da Fonseca et Leão présentait une gale ayant 5 années d'évolution.

Chez certains malades, le prurit n'est pas intense et alors l'absence de traitement et le défaut de propreté contribuent à prolonger la maladie; il se produit alors une irritation chronique de l'épiderme.

L'aspect d'érythème, très accentué chez quelques malades, tel que celui de Dubreuilh, est dù à la vaso-dilatation signalée à plusieurs reprises au cours de notre étude.

En terminant, nous devons déclarer que, dans cette observation, nous n'avons trouvé aucune lésion d'aspect lépreux.

\section{Conchusions}

L'auteur décrit un cas de gale norvégienne ou croûteuse, diffusément répandue chez une femme métisse.

Les altérations cutanées consistent en parakératose, œdème du derme et de l'épiderme.

Les vaisseaux du derme sont dilatés avec infiltration cellulaire périphérique.

On n'a pas observé de lésions lépreuses.

Les parasites sont abondants et à toutes les phases de leur développement.

L'auteur conclut à l'identité des acariens de la gale commune et de la gale norvégienne.

Laboratoire d'Anatomie pathologique de la Faculté de Médecine de Rio-de-Janeiro.

(Directeur : Prof. Leitâo da Cunha). 